\title{
A novel noninvasive method for measuring fractional flow reserve through three-dimensional modeling
}

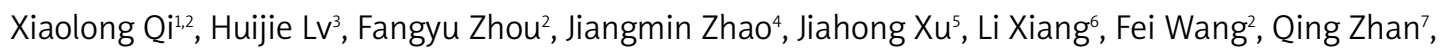
Jinfa Jiang ${ }^{5}$, Junjie $\mathrm{XiaO}^{1}$

1Experimental Center of Life Sciences and Regeneration Lab, School of Life Science, Shanghai University, Shanghai, China

2Shanghai Tongji Hospital, Tongji University School of Medicine, Shanghai, China

${ }^{3}$ Division of Mathematics, Tongji University, Shanghai, China

${ }^{4}$ Department of Radiology, Shanghai Third People's Hospital, Shanghai Jiaotong

University School of Medicine, Shanghai, China

${ }^{5}$ Department of Cardiology, Tongji Hospital, Tongji University School of Medicine,

Shanghai, China

${ }^{6}$ Department of General Surgery, Second Xiangya Hospital, Central South University, Changsha, China

7Department of Neurology, Tongji Hospital, Tongji University School of Medicine,

Shanghai, China

Submitted: 4 September 2012

Accepted: 16 September 2012

Arch Med Sci 2013; 9, 3: 581-583

DOI: 10.5114 /aoms.2013.35020

Copyright (c) 2013 Termedia \& Banach

Coronary stenosis with lumen diameter reduction greater than $50 \%$ is recognized as coronary artery disease (CAD) [1-4]. Fractional flow reserve (FFR) is an epicardial lesion-specific parameter to determine the functional coronary stenosis, which is determined by pressure difference and resistance [5-8]. Previous studies have demonstrated that FFR guided percutaneous coronary intervention $(\mathrm{PCI})$ could improve outcomes compared with anatomical invasive coronary angiography (ICA) guided $\mathrm{PCI}$ [6-10]. This study aims to overcome the deficiencies of invasive FFR and create a novel noninvasive FFR (FFR $\left.{ }_{\text {ni }}\right)$.

A 70-year-old female patient, diagnosed with CAD, stable angina pectoris, cardiac function III, essential hypertension III (very high-risk group), with blood pressure of $140 / 85 \mathrm{~mm} \mathrm{Hg}$, was enrolled in as a pilot study.

Coronary computed tomography angiography (CCTA) was performed using multi-detector computed tomography scanners (Lightspeed 16 Pro). Original images were spilt into thin layers and directly exported into imaging control software MIMICS and processed to form an image sequence. Three-dimensional (3D) geometric models of the narrow coronary arteries including the right coronary artery (RCA), left anterior descending coronary (LAD) and left circumflex artery (LCX) were reconstructed and exported. Then, the geometric model was meshed with 3D Flotran elements in ANSYS software. Finite element analysis (FEA) was applied to analyze the velocity and pressure distribution of selected coronary arteries. Given boundary conditions including average velocity measured by transthoracic Doppler echocardiography with an ultrasound system (Sequoia C256) were applied in an inlet of 3D model. By setting the proper iteration time, the calculation went smoothly. ICA, as the "gold standard", was performed with standard techniques.

Invasive coronary angiography indicated that there was around $80 \%$ stenosis in the proximal RCA (Figure $1 \mathrm{~A}$ ). Mild (about 30\%) stenosis in the proximal LAD and a diffuse lesion of the LCX (the narrowest was $80 \%$ )

\author{
Corresponding author: \\ Dr. Junjie Xiao \\ Experimental \\ Center of Life Sciences \\ and Regeneration Lab \\ School of Life Science \\ Shanghai University \\ 333 Nan Chen Road \\ Shanghai 200444, China \\ Phone: +86 2166138131 \\ Fax: +86 2166138131 \\ E-mail: junjiexiao@live.cn, \\ junjiexiao@shu.edu.cn
}



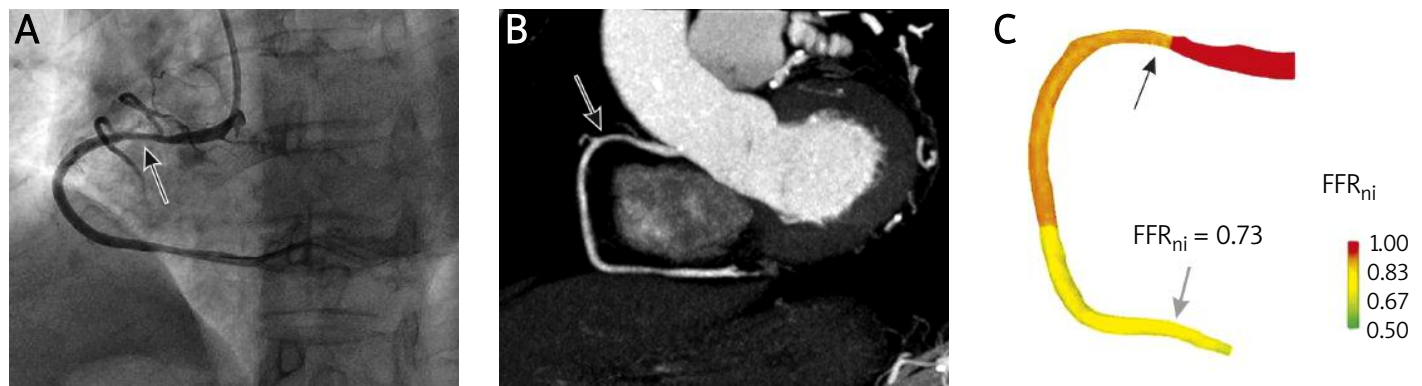

Figure 1. Anatomically obstructive stenosis of right coronary artery (RCA) with a lesion causing ischemia. A - Invasive coronary angiography indicates that stenosis is about $80 \%$ in the proximal RCA (black arrow). B - Multi-planar reformat of coronary computed tomography angiography demonstrates moderate (around 60\%) obstructive steno-

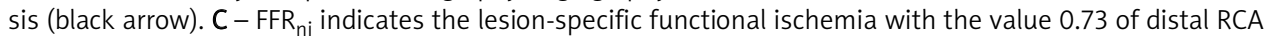
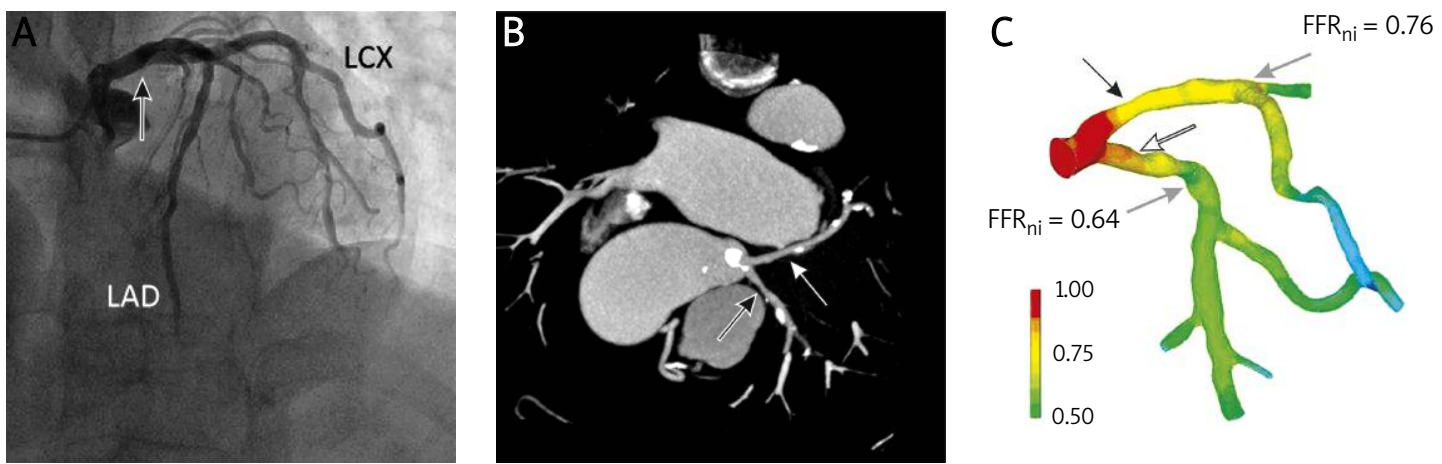

Figure 2. Anatomically obstructive stenosis of left anterior descending coronary (LAD) and left circumflex artery (LCX) with/without functional ischemia. A - Invasive coronary angiography indicates that there exist 30\% stenosis in proximal LAD (black arrow) and a diffuse lesion (narrowest point around 80\% stenosis) in LCX. B - Multi-planar reformat from coronary computed tomography angiography shows about $30 \%$ stenosis in proximal LAD (black arrow) and diffuse stenosis in LCX (white arrow). C - Lesion-specific functional ischemia is indicated with FFR $\mathrm{ni}_{\text {i }}$ value 0.76 , 0.64 in the distal stenosis of LAD and LCX, respectively

could also be quantified (Figure 2 A). Additionally, the coronal section of CCTA images demonstrated moderate (around 60\%) obstructive stenosis in the proximal RCA (Figure $1 \mathrm{~B}$ ). The cross-sectional images indicated $30 \%$ stenosis in the proximal and first branch segment of the LAD and diffuse patchy calcified plaques in the LCX leading to intermediate $(50 \%)$ stenosis (Figure 2 B). The diagnostic performance of $\mathrm{FFR}_{\mathrm{ni}}$ was generally consistent with the results of ICA and CCTA. The values of proximal RCA, LAD and distal LCX were $0.73,0.76$ and 0.64 , respectively, which suggested the severity of lesion-specific functional ischemia in distal myocardium with 0.75 as the cutoff value (Figures 1 C and 2 C).

Recently, FFR computed from CCTA $\left(F_{F R}\right)$ was reported. A good correlation between $\mathrm{FFR}_{\mathrm{CT}}$ and invasive FFR was certified through a randomized clinical controlled trial on 159 vessels in 103 patients [11]. The differences in the calculation process of $F F R_{C T}$ and $F F R_{n i}$ in our study mainly reflect the following factors. During $F_{F R}$ calculation, coronary flow and pressure are unknown. A method to couple lumped parameter models of the microcirculation to the outflow boundaries of the 3D model was adopted. As a result of the cumbersome workload, it takes approximately $5 \mathrm{~h}$ /exam. We utilized FEA of the Flotran CFD module to solve the hemodynamic calculation under given boundary conditions. Therefore, it can greatly reduce the computation time to $3 \mathrm{~h}$ /exam. The more relaxed equipment requirements and faster inspection time guarantee potential clinical application of $\mathrm{FFR}_{\mathrm{ni}}$.

Fractional flow reserve is a well-evaluated functional index to assess the ischemic significance of coronary lesions, helping making the decision of revascularization [12-14]. Nevertheless, the invasiveness and costliness are two major reasons restricting its further application. Finite element analysis and CFD over digital 3D modeling were applied in this pilot study and created a novel method to evaluate functional coronary stenosis by FFR $_{\text {ni }}$, which showed good consistency with ICA and CCTA. The superiority of no invasiveness and cost-effectiveness establishes the foundation of FFR ${ }_{n i}$ for its further applications in clinical practice. However, a large randomized clinical controlled trial assessed by FFR $_{\text {ni }}$ compared with invasive FFR is urgently needed.

\section{Acknowledgments}

Xiaolong Qi and Huijie Lv contributed equally to this work. 
This work was supported by grants from the National Natural Science Foundation of China (81200669, to Junjie Xiao), the Innovation Program of Shanghai Municipal Education Commission (13YZ014 to Junjie Xiao), Innovation Foundation of Shanghai University (to Junjle Xias), a Research Training Grant for undergraduates of Tongji University (1500219050, to Xiaolong Qi), and partially by leading Academic Discipline Project of Shanghai Municipal Education Commission "Molecular physiology".

\section{References}

1. Meijboom WB, Van Mieghem CA, van Pelt N, et al. Comprehensive assessment of coronary artery stenosis. J Am Coll Cardiol 2008; 52: 636-43.

2. Tobis J, Azarbal B, Slavin L. Assessment of intermediate severity coronary lesions in the catheterization laboratory. J Am Coll Cardiol 2007; 49: 839-48.

3. Christou MA, Siontis GC, Katritsis DG, loannidis JP. Metaanalysis of fractional flow reserve versus quantitative coronary angiography and noninvasive imaging for evaluation of myocardial ischemia. Am J Cardiol 2007; 99: 450-6.

4. Costa MA, Shoemaker S, Futamatsu H, et al. Quantitative magnetic resonance perfusion imaging detects anatomic and physiologic coronary artery disease as measured by coronary angiography and fractional flow reserve. J Am Coll Cardiol 2007; 50: 514-22.

5. Rieber J, Huber A, Erhard I, et al. Cardiac magnetic resonance perfusion imaging for the functional assessment of coronary artery disease: a comparison with coronary angiography and fractional flow reserve. Eur Heart J 2006; 27: 1465-71.

6. Lindstaedt M, Mügge A. Myocardial fractional flow reserve - its role in guiding $\mathrm{PCl}$ in stable coronary artery disease. Herz 2011; 36: 410-6.

7. Koo BK. Physiologic evaluation of bifurcation lesions using fractional flow reserve. J Interv Cardiol 2009; 22: 110-3.

8. Puymirat É, Muller O, Sharif F, et al. Fractional flow reserve: concepts, applications and use in France in 2010. Arch Cardiovasc Dis 2010; 103: 615-22.

9. Schelbert HR. FFR and coronary flow reserve: friends or foes. JACC Cardiovasc Imaging 2012; 5: 203-6.

10. Hamilos M, Peace A, Kochiadakis G, et al. Fractional flow reserve: an indispensable diagnostic tool in the cardiac catheterisation laboratory. Hellenic J Cardiol 2010; 51: 133-41.

11. Koo BK, Erglis A, Doh JH, et al. Diagnosis of ischemiacausing coronary stenosis by noninvasive fractional flow reserve computed from coronary computed tomographic angiograms. J Am Coll Cardiol 2011; 58: 1989-97.

12. Uznanska-Loch B, Plewka M, Peruga JZ, et al. Non-invasive detection of concomitant coronary artery anomaly and atherosclerotic coronary disease using transthoracic Doppler echocardiography. Arch Med Sci 2012; 8: 162-5.

13. De Bruyne B, Sarma J. Fractional flow reserve: a review. Heart 2008; 94: 949-59.

14. Melikian N, De Bondt P, Tonino P, et al. Fractional flow reserve and myocardial perfusion imaging in patients with angiographic multivessel coronary artery disease. JACC Cardiovasc Interv 2010; 3: 307-14. 